\title{
SIMULAÇÃO DO ESCOAMENTO IMISCÍVEL DE UM JATO DE ÓLEO EM MEIO SUBAQUÁTICO PARA O INTERIOR DE UM RESERVATÓRIO DE COLETA
}

\author{
J. de B. BIEHL ${ }^{1}$, M. B. QUADRI ${ }^{1}$, M. C. dos $\operatorname{SANTOS}^{2}$ e N. R. ULIANA ${ }^{1}$ \\ ${ }^{1}$ Universidade Federal de Santa Catarina, Departamento de Engenharia Química e \\ Engenharia de Alimentos \\ ${ }^{2}$ CENPES/PETROBRAS \\ E-mail para contato: mbq1959@hotmail.com
}

\begin{abstract}
RESUMO - Vazamentos de petróleo no fundo do mar, tanto em situações de explotação como de transporte provocam perdas de natureza econômica, ambiental e social. Para minimizar esses impactos, o desenvolvimento de soluções inovadoras é necessário e devem priorizar a captura do óleo antes da sua ascensão e espalhamento na superfície. Estudos experimentais e de simulação dos fenômenos envolvidos são imprescindíveis até que se possa chegar ao projeto de um equipamento de captura apto a operar em condições de campo. Foram realizadas simulações do escoamento de um jato de óleo através de um bocal de coleta para estudar o comportamento da ascensão do óleo para um dispositivo submerso que o colete e armazene sem os prejuízos do seu espalhamento na superfície Ao se testar diferentes velocidades de injeção do óleo, bem como diferentes diâmetros do bocal de coleta, foi constatada a viabilidade da operação, além de se perceber que é possível minimizar a quantidade de água arrastada pelo jato de óleo que entra no dispositivo.
\end{abstract}

\section{INTRODUÇÃO}

A atividade de extração petrolífera offshore apresenta aspectos de difícil equacionamento devido à complexidade do processo e o risco de acidentes envolvendo vazamentos, os quais costumam ser de grandes proporções e de difícil quantificação (YAPA et al., 1999). Os métodos existentes de contenção de vazamentos se mostram ineficientes do ponto de vista ambiental e financeiro (HENNING, 1993), uma vez que apenas contemplam a retirada do óleo da água do mar e não a sua recuperação (MIRANDA, 2011).

No transporte de petróleo por dutos existem variações de pressão no sistema de escoamento, resultantes de diferentes elevações do terreno, de velocidade de escoamento e de fricção, além de uma variedade de conexões, curvas e variações abruptas de área. Esses fatores podem causar rupturas nos dutos, gerando vazamentos (YUE et al., 2006). Em um vazamento de petróleo no oceano a descarga inicial muitas vezes apresenta um comportamento fluidodinâmico multifásico característico (PALADINO, 2000). A caracterização desse comportamento depende de fatores como a taxa de vazamento, o tamanho da ruptura, das propriedades do petróleo e das 
condições do ambiente (SANTOS et al., 2003). O potencial mais importante que contribui para a elevação do óleo proveniente de um vazamento em alto mar corresponde à força de flutuação, sendo essa a diferença entre a força gravitacional e a força de empuxo (WÖRNER, 2003). Nesse contexto, esse trabalho é direcionado para estudos que objetivam de simular o comportamento fluidodinâmico de sistemas bifásicos água/óleo, que são elementos-chave para a reprodução da fenomenologia desses eventos em condições de campo relacionadas à captura de óleo proveniente de um vazamento (MARIANO, 2008).

\section{MATERIAL E MÉTODOS}

Os estudos de simulação foram desenvolvidos nos softwares COMSOL Multiphysics $4.3 a$ e no Wolfram Mathematica v.8. Foram consideradas as principais propriedades físicas dos fluidos do estudo (óleo de soja e água), como: massa específica, viscosidade e tensão interfacial, além dos efeitos de pressão próprios das condições operacionais, bem como as instabilidades da interface (ANDRADE, 2009).

O COMSOL possui ferramentas de CFD aplicáveis aos escoamentos bifásicos que permitem considerar os efeitos de convecção - difusão ao nível das interfaces do sistema água/óleo. Nesta abordagem, assume-se escoamento laminar, isotérmico e domínio bidimensional axissimétrico para modelar a ascensão do óleo. A discretização das equações diferenciais que regem o fenômeno é feita através do método dos elementos finitos (CHEN and YAPA, 2004), empregando-se uma discretização de segunda ordem para o campo de velocidades e de primeira ordem para o campo de pressões. Foi desenvolvido no COMSOL um modelo de um jato vertical ascendente passando por um tubo submerso, onde não haveria contra fluxo de água, correspondente a etapa inicial em que o dispositivo estaria recebendo óleo e água simultaneamente. Já no Mathematica, procurou-se desenvolver um modelo simplificado para o estudo do jato em um bocal de recepção. Este modelo foi ajustado aos resultados obtidos com o COMSOL. Na Figura 1 esquematiza-se o domínio e condições de contorno adotadas para a solução do problema.

Em presença da força gravitacional, os campos de velocidades e pressão para um dos fluidos de um sistema bifásico em regime laminar incompressível são descritos pela Equações 1 de Navier-Stokes - e 2 da continuidade da massa, que em notação vetorial são dadas por, respectivamente (GIRAULT and RAVIART, 1979):

$$
\begin{aligned}
& \rho \frac{\partial \mathbf{u}}{\partial t}+\rho(\mathbf{u} \cdot \nabla) \mathbf{u}=\nabla \cdot\left[-p \boldsymbol{I}+\mu\left(\nabla \mathbf{u}+\nabla \mathbf{u}^{T}\right)-\frac{2}{3} \mu(\nabla \cdot \mathbf{u}) \boldsymbol{I}\right]+\rho g+\gamma \\
& \nabla \cdot \mathbf{u}=0
\end{aligned}
$$

Onde $\rho$ é a densidade $\left(\mathrm{kg} / \mathrm{m}^{3}\right)$, u é o vetor velocidade $(\mathrm{m} / \mathrm{s}), t$ é o tempo, $p$ é a pressão $(\mathrm{Pa})$, 
$\boldsymbol{I}$ é o sistema de vetores unitários, $T$ é a operação transposta da matriz, $\boldsymbol{g}$ é a aceleração gravitacional $\left(\mathrm{m} / \mathrm{s}^{2}\right), \mu$ é a viscosidade dinâmica (mPa.s) e $\gamma$ é a tensão interfacial $(\mathrm{mN} / \mathrm{m})$.

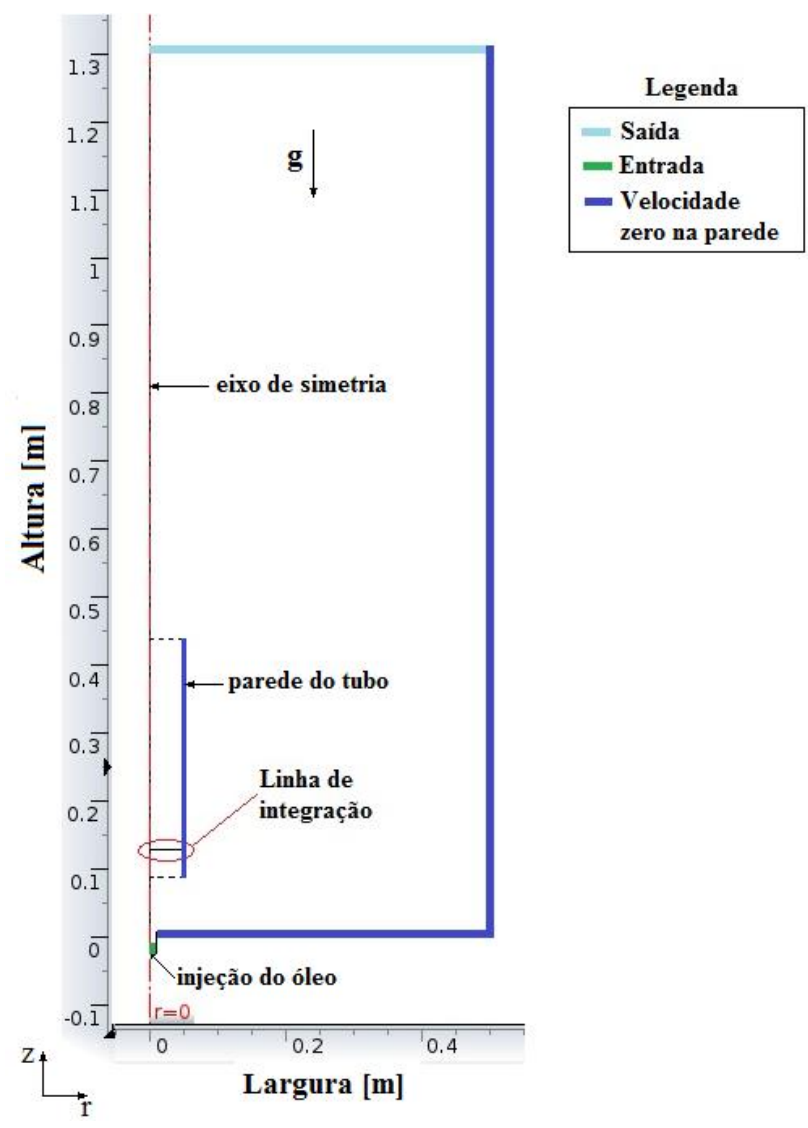

Figura 1 - Domínio e condições de contorno consideradas no problema

\subsection{Modelo phase-field}

Para modelar o fluxo de dois diferentes líquidos imiscíveis, onde a posição exata da interface é de interesse, o modelo proposto por Cahn and Hilliard (1958) está implementado no software COMSOL sob a denominação de phase-field. Esse modelo considera as densidades e viscosidades dos fluidos, inclui o efeito da tensão superficial e gravidade. A dinâmica da interface é descrita pela Equação 3:

$$
\frac{\partial \phi}{\partial t}+\mathbf{u} \cdot \nabla \phi=\nabla \cdot \delta \nabla G
$$

Onde $\phi$ é a variável campo de fase adimensional sendo que as frações de volume dos componentes do fluido são dadas por $(1+\phi) / 2$ e $(1-\phi) / 2 . \delta$ é a mobilidade da interface $\left(\mathrm{m}^{3} \cdot \mathrm{s} / \mathrm{kg}\right)$ e $G$ é o potencial químico $(\mathrm{Pa})$. 


\subsection{Modelo de escoamento simplificado}

O problema do fluxo de água arrastada por um jato de óleo ao passar através do tubo/bocal de recepção aberto também pode ser abordado de forma simplificada. Seguindo a teoria apresentada por Bird et al (2002) e adaptando-se para um domínio axissimétrico onde a força de flutuação induzida pela diferença de densidades dos fluidos atua apenas na fase óleo, que está imersa na fase água, um balanço diferencial de momento para as fases óleo e água leva, respectivamente, às seguintes equações:

$$
\begin{aligned}
& \frac{1}{r} \frac{d\left(r \tau_{o r z}\right)}{d r}=k_{o w}\left(-\frac{\Delta P_{o}}{H}-\left(\rho_{w}-\rho_{o}\right) g\right) \\
& \frac{1}{r} \frac{d\left(r \tau_{w_{r z}}\right)}{d r}=-\frac{\Delta P_{w i}}{H}
\end{aligned}
$$

Onde $\tau_{\text {orz }}$ e $\tau_{w r z}$ são as tensões no plano $r z$ para as fases óleo e água, respectivamente; $-\Delta P_{o} / H$ é o gradiente de pressão efetivo na fase óleo na direção vertical (z) negativo para cima no interior do tubo cilíndrico de altura $H \mathrm{e}-\left(\rho_{w}-\rho_{o}\right) g$ o termo de flutuação; $k_{\text {ow }}$ é um fator adimensional de penetrabilidade; $-\Delta P_{\text {wi }} / H$, é o gradiente de pressão efetivo negativo para cima induzido na fase água devido aos efeitos de entrada do óleo no tubo; $\rho_{w}$ e $\rho_{o}$ as densidades da água e do óleo, respectivamente; e $\boldsymbol{g}$ a aceleração da gravidade $\left(\mathrm{m} / \mathrm{s}^{2}\right)$.

Devido à abordagem axissimétrica adotada, a componente de translação $w$ na direção $\theta$ e as correspondentes deformações cisalhantes são nulas.

A integração das Equações (4) e (5), considerando as condições: em $r=r_{l}$, na interface água/óleo, tem-se $\tau_{o r z}=\tau_{w r z}$ e também $v_{o z}=v_{w z}$; em $r=0$, onde se encontra o eixo de simetria, tem-se $\frac{d v_{0 z}}{d r}=0$; e por fim, na parede do tubo, em $r=r_{T}$ (raio do tubo) tem-se condição de parede (no slip), ou seja, $v_{w z}=0$. Após calculadas as constantes de integração, pode-se então determinar os perfis de velocidade das fases óleo e água, segundo as Equações 6 e 7:

$$
\begin{aligned}
& v_{o z}=-\frac{r^{2}}{4 \mu_{o}} k_{o w}\left(\frac{-\Delta P_{o}}{H}-\left(\rho_{w}-\rho_{o}\right) g\right)-C_{o 2} \\
& v_{w z}=-\frac{r^{2}}{4 \mu_{w}}\left(\frac{-\Delta P_{w i}}{H}\right)-\frac{C_{w 1} \ln r}{\mu_{w}}-C_{w 2}
\end{aligned}
$$

As equações supracitadas foram implementadas no Wolfram Mathematica $v .8$ de modo a ajustar o modelo simplificado aos resultados obtidos com o COMSOL Multiphysics $4.3 \mathrm{a}$ para as diferentes situações testadas. 


\section{RESULTADOS E DISCUSSÃO}

Foi simulado o escoamento laminar do óleo através de um tubo cilíndrico com $35,0 \mathrm{~cm}$ de altura, com diâmetros de $5,7 \mathrm{~cm}, 7,7 \mathrm{~cm}$ e $9,7 \mathrm{~cm}$ e velocidades de injeção de óleo de $0,35 \mathrm{~m} / \mathrm{s}$, $0,7 \mathrm{~m} / \mathrm{s}$ e $1,05 \mathrm{~m} / \mathrm{s}$. O diâmetro do jato de injeção de óleo foi de $1,1 \mathrm{~cm}$, o mesmo dos experimentos. Aqui foi estudado o comportamento do óleo ao passar pelo tubo de recepção em direção ao balão em processo de desdobramento. A distância do ponto de injeção até a entrada do tubo foi de $8,0 \mathrm{~cm}$.

O modelo phase-field foi utilizado para simular o escoamento bifásico água/óleo em regime transiente. As propriedades dos fluidos simulados são as mesmas do óleo e da água utilizados nos experimentos. O parâmetro que controla a espessura da interface $\varepsilon_{p f}$ foi assumido como tpf.hmax/4, ou seja, um quarto do tamanho do maior elemento da malha. O parâmetro de ajuste de mobilidade requerido foi inserido com o valor de $1,0 \mathrm{~m} . \mathrm{s} / \mathrm{kg}$ e o valor da derivada $\phi$ da energia livre externa $(\partial f / \partial \phi)$ foi mantido no valor padrão de $0,0 \mathrm{~J} / \mathrm{m}^{3}$. A velocidade do escoamento tangencial nas paredes foi considerada nula, condição de contorno chamada de no slip. Pressão de 1 atm (1,013 MPa) foi considerada na parte superior do domínio (saída). Adicionalmente, um perfil hidrostático inicial de pressão foi adotado de forma a considerar o peso da coluna de água de $1,3 \mathrm{~m}$. O tempo total simulado foi de 40 segundos.

A malha utilizada foi refinada sucessivamente na região do escoamento para que houvesse maior precisão nos cálculos. Quando foi testada uma malha considerada grosseira, com 9.719 elementos, não se obteve um bom resultado. Refinando-se a partir daí, agora com 12.322 elementos, o escoamento se apresentou fisicamente coerente, mas com a interface ainda pouco definida. A partir de 23.008 elementos, as simulações começaram a apresentar melhor convergência e os resultados ficaram menos dependentes do tamanho da malha. Ao final foram utilizados 64.421 elementos triangulares, buscando-se atender a uma precisão não inferior a $10^{-3}$ nos cálculos. O Pardiso solver, que trabalha com operações de substituição direta, foi escolhido para resolver o sistema de equações lineares resultantes da discretização de equações diferenciais. A Figura 2 a seguir mostra em detalhe um plano de corte axial na região do tubo exemplificando um resultado de simulação da coluna de óleo (região vermelha) que passa por um tubo de 9,7 cm de diâmetro (cuja parede corresponde ao retângulo branco) e com velocidade de injeção do óleo de $1,05 \mathrm{~m} / \mathrm{s}$. Foi constatado que quanto maior a velocidade de injeção de óleo, maior o diâmetro da região afetada pelo jato devido ao aumento do efeito de difusão junto à interface. A região azul é correspondente à água e a interface se situa na região de transição de cores com $\phi=0,5$.

Aos 2,4 segundos de simulação, constata-se que o escoamento se estabilizou. Neste caso o diâmetro do jato permaneceu estável após esse tempo, com valor de $1,95 \mathrm{~cm}$, quase duas vezes maior que o diâmetro do bico de injeção. De um modo geral, a estabilização do escoamento não ultrapassou 6,4 segundos em nenhum dos casos estudados. A principal diferença observável entre esses casos foi o diâmetro do jato de óleo, que variou segundo a velocidade de injeção. A Tabela 1 mostra os valores do diâmetro do jato de óleo na entrada/saída do tubo para cada um dos diâmetros de tubo estudados e as respectivas velocidades de injeção de óleo aplicadas. 

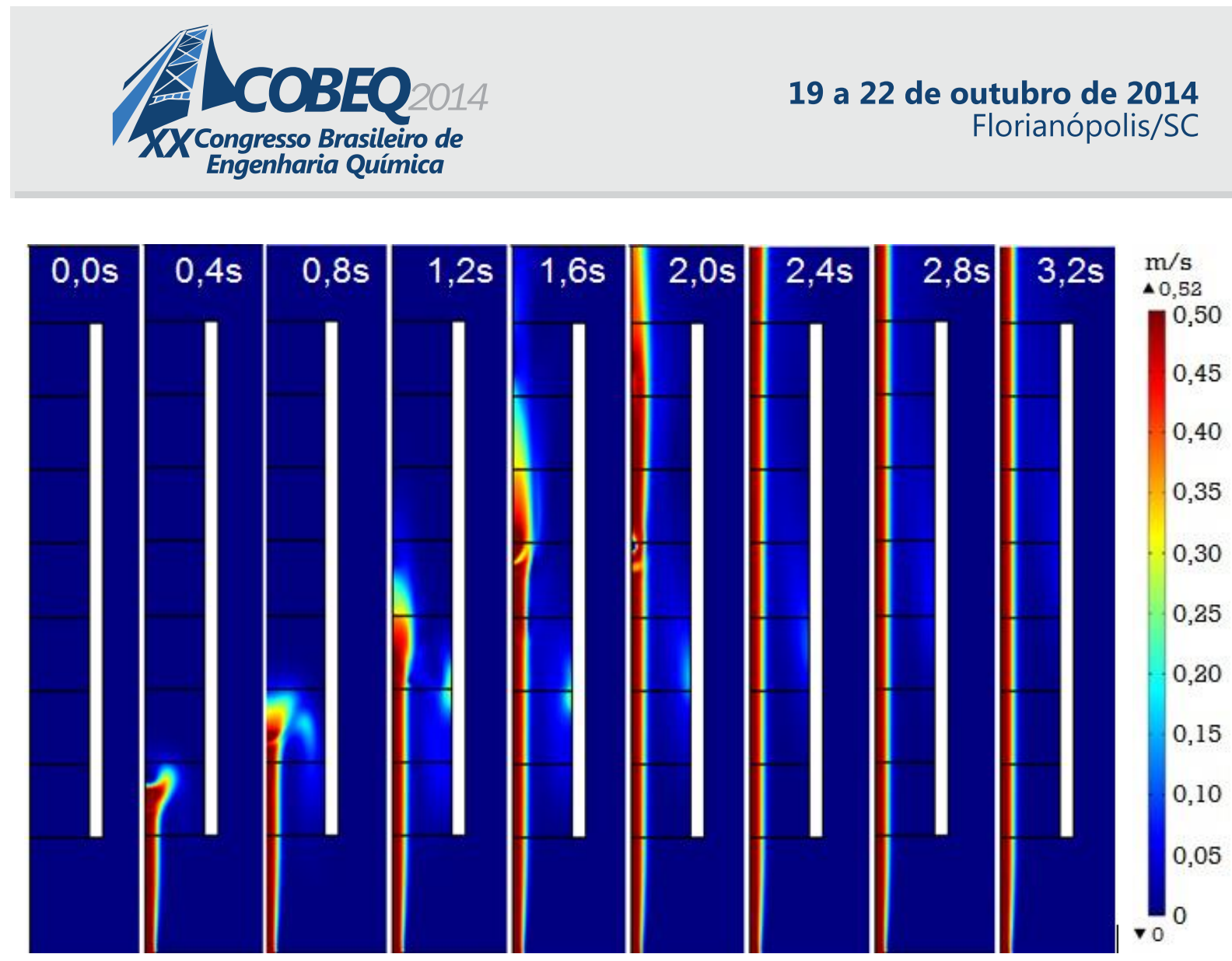

Figura 2 - Sequência de quadros cobrindo a passagem do jato de óleo através tubo até a estabilização do fluxo laminar para a velocidade de injeção de $1,05 \mathrm{~m} / \mathrm{s}$ e diâmetro do tubo de 9,7 $\mathrm{cm}$

Tabela 1 - Diâmetro do jato para cada velocidade de injeção na entrada e saída do tubo

\begin{tabular}{cccc}
\hline Velocidade $\backslash$ diâmetro tubo & $5,7 \mathrm{~cm}$ & $7,7 \mathrm{~cm}$ & $9,7 \mathrm{~cm}$ \\
\hline $0,35 \mathrm{~m} / \mathrm{s}$ & $1,42 / 0,90 \mathrm{~cm}$ & $1,46 / 1,05 \mathrm{~cm}$ & $1,46 / 0,88 \mathrm{~cm}$ \\
$0,70 \mathrm{~m} / \mathrm{s}$ & $1,76 / 1,70 \mathrm{~cm}$ & $1,79 / 1,69 \mathrm{~cm}$ & $1,80 / 1,64 \mathrm{~cm}$ \\
$1,05 \mathrm{~m} / \mathrm{s}$ & $1,90 / 2,02 \mathrm{~cm}$ & $1,923 / 2,0 \mathrm{~cm}$ & $1,94 / 1,95 \mathrm{~cm}$ \\
\hline
\end{tabular}

Para todos os diâmetros dos tubos, na velocidade de $0,35 \mathrm{~m} / \mathrm{s}$, devido à baixa vazão de óleo, ocorreu um estreitamento do jato de óleo ao longo da altura, partindo de um diâmetro maior na entrada do tubo até outro menor na saída. Na velocidade de injeção de $0,70 \mathrm{~m} / \mathrm{s}$, o jato permanece praticamente com mesmo diâmetro enquanto passa pelo tubo.

\subsection{Estudo dos perfis de velocidade no bocal de coleta}

A Figura 3 mostra os perfis de velocidade ao longo dos oito segmentos horizontais em preto (b1 a b8) para a velocidade de injeção de óleo de $1,05 \mathrm{~m} / \mathrm{s}$ e diâmetro de tubo de $9,7 \mathrm{~cm}$ aos 40 segundos de simulação. 

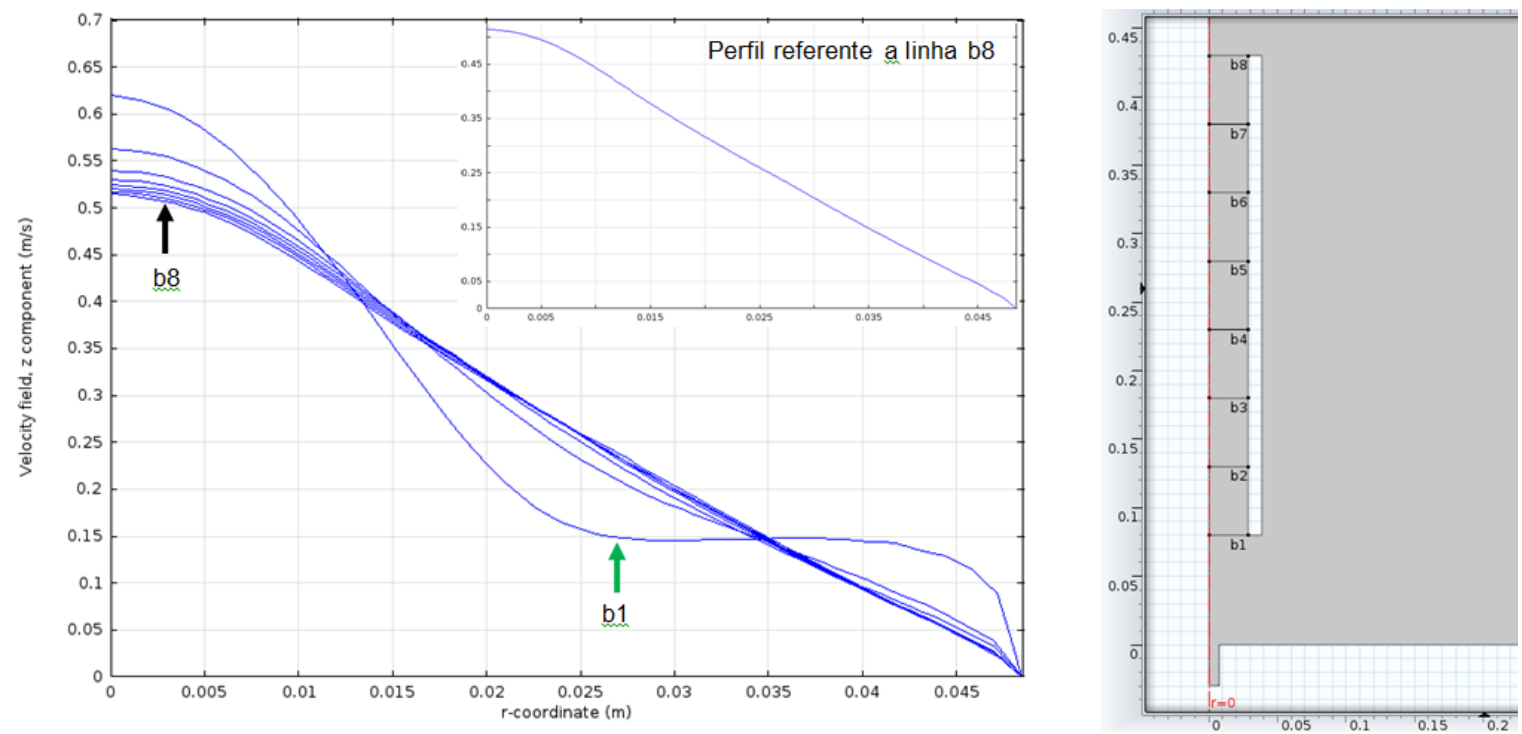

Figura 3 - Perfis de velocidade ao longo das 8 linhas (b1 a b8) para a velocidade de 1,05 m/s no tubo de diâmetro $9,7 \mathrm{~cm}$ no regime laminar aos 40 segundos de simulação

Em todos os estudos, obteve-se uma velocidade máxima no centro da geometria (raio igual a zero), e um decréscimo a partir da mesma ao longo do raio. Como esperado, quanto maior a velocidade de injeção do óleo, maiores as velocidades de ascensão do jato através do tubo. A velocidade máxima atingida pelo óleo no eixo de simetria não ultrapassou a velocidade injetada, evidenciando o efeito de arraste sobre o óleo exercido pelas forças de cisalhamento e de forma (drag forces). O escoamento pode gerar emulsões com comportamento Newtoniano quando o número de Reynolds ultrapassa 2.000 (ROSSI and MORALES, 2004).

O modelo simplificado para a coluna de óleo ascendente foi implementado no Wolfram Mathematica $v .8$ e ajustado aos resultados obtidos com as simulações realizadas no COMSOL. Os perfis de velocidade foram ajustados segundo a regressão dos valores do parâmetro penetrabilidade $\left(k_{o w}\right)$ e do gradiente de pressão $-\Delta P_{w i} / H$. A penetrabilidade engloba os efeitos de arraste sobre o óleo e forças de tensão interfacial de forma a incluir aspectos fenomenológicos inerentes ao problema. Com base nas simulações realizadas no COMSOL, um valor médio aceitável para $k_{o w}$, na ausência de contra fluxo, seria $2,75 \times 10^{-2}$, mas sujeito a variações segundo as dimensões geométricas envolvidas e a intensidade do jato de óleo. Em todos os casos estudados, $-\Delta P_{\text {wi }} / H$ ficou abaixo de $2 \mathrm{~Pa} / \mathrm{m}$. É necessário considerar um valor de fechamento para viscosidade média $\left(\mu_{m}\right)$ na interface água/óleo, justificada por um grau de mistura, que garantirá a continuidade do perfil de velocidades. Os demais parâmetros foram todos tomados tais e quais aqueles utilizados nas simulações com o COMSOL.

A Figura 4 compara os resultados dos perfis de velocidade para injeção a 1,05 m/s obtidos no modelo simplificado, com aqueles do COMSOL, para o diâmetro de tubos de $9,7 \mathrm{~cm}$. Para as outras velocidades de injeção, ocorreu comportamento similar. 


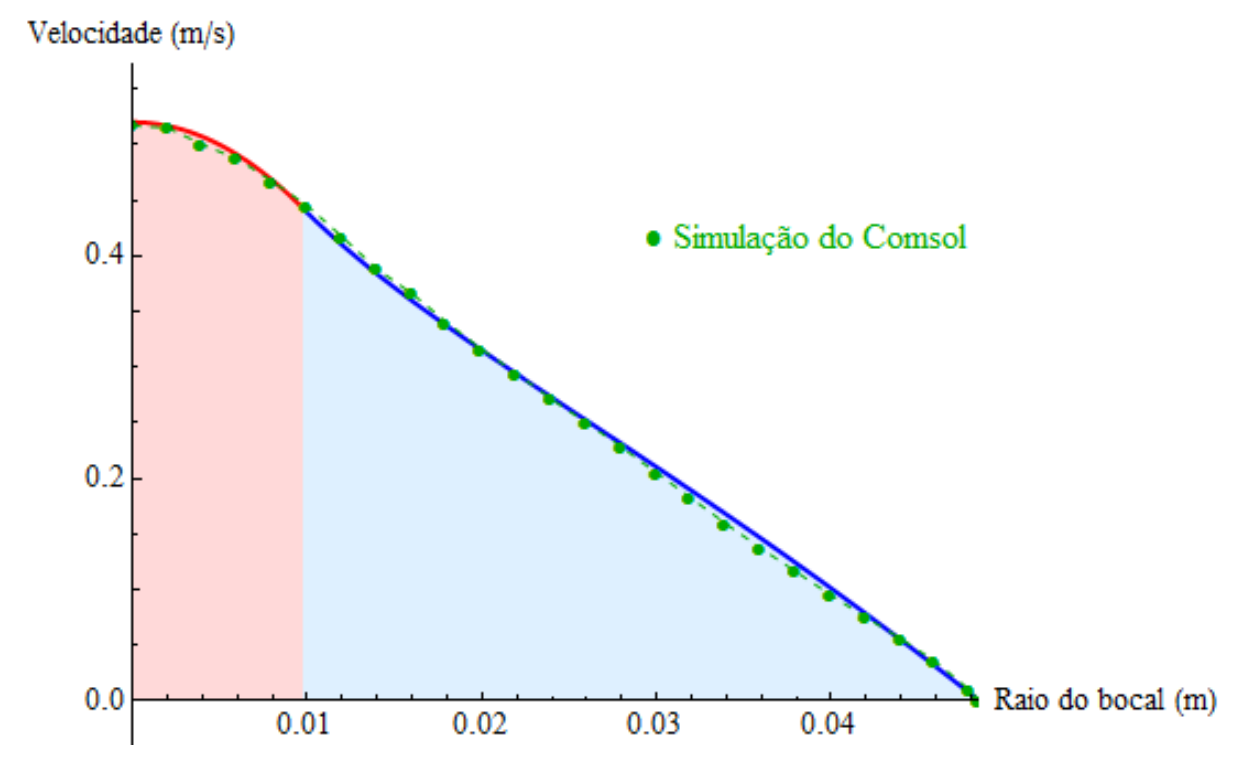

Figura 4 - Perfil de velocidade de 1,05 m/s nos diâmetro de tubo de $9,7 \mathrm{~cm}$

De uma forma geral, pode-se dizer que o modelo simplificado pôde ser satisfatoriamente ajustado aos resultados de simulação do COMSOL. Por outro lado, os gradientes elevados de velocidade de ascensão na direção do raio junto à parede do tubo não puderam ser bem representados pelo modelo simplificado. De fato, os efeitos de entrada e do escoamento do lado de fora do tubo não são considerados na abordagem simplificada, o que pode ser o motivo para tais discrepâncias. Percebe-se que ao se ajustar o modelo simplificado, as vazões de óleo são sempre menores do que as produzidas nas simulações do COMSOL.

\section{CONSIDERAÇÕES FINAIS}

O estudo fenomenológico de escoamento bifásico água/óleo em situações vazamento em águas profundas é crucial para que se tenha uma ideia do que possivelmente ocorrerá em um caso real. Estudos têm sido desenvolvidos para que a fluidodinâmica da ascensão de óleo em meio subaquático seja devidamente representada.

Os resultados obtidos neste trabalho ajudam a descrever e antecipar o comportamento de um jato laminar de óleo que deverá preencher um dispositivo de coleta, tal como um reservatório submerso. Nesse sentido, estudos considerando a ampliação de escala serão indispensáveis para o desenvolvimento de um equipamento com a função de fazer a referida coleta de óleo.

Ambos os modelos apresentados se mostraram adequados frente ao que se conhece dos fenômenos físicos envolvidos nesse tipo de sistema. Além disso, a observância de critérios de estabilidade, convergência numérica e unicidade da solução permitem atribuir o necessário grau de credibilidade aos resultados apresentados. 


\section{NOMENCLATURA}

\begin{tabular}{|c|c|c|c|c|}
\hline Constante & $(-)$ & $\mathrm{z}$ & Coordenada axial & $\mathrm{m}$ \\
\hline Energia livre externa & $\mathrm{J} / \mathrm{m}^{3}$ & \multicolumn{3}{|c|}{ Letras gregas } \\
\hline Aceleração gravitacional & $\mathrm{m} / \mathrm{s}^{2}$ & $\gamma$ & Tensão interfacial & $\mathrm{N} / \mathrm{m}$ \\
\hline Potencial químico & $\mathrm{Pa}$ & $\delta$ & Mobilidade da interface & $\mathrm{m}^{3} \cdot \mathrm{s} / \mathrm{kg}$ \\
\hline Altura & $\mathrm{m}$ & $\varepsilon$ & Comprimento capilar & $\mathrm{m}$ \\
\hline Sistema de vetores unitários & $(-)$ & $\lambda$ & Densidade de energia da mistura & $\mathrm{N}$ \\
\hline Penetrabilidade & $(-)$ & $\mu$ & Viscosidade & Pa.s \\
\hline Pressão & $\mathrm{Pa}$ & $\rho$ & Densidade & $\mathrm{kg} / \mathrm{m}^{3}$ \\
\hline Coordenada radial & $\mathrm{m}$ & $\tau$ & Tensão de cisalhamento & $\mathrm{N} / \mathrm{m}^{2}$ \\
\hline Tempo & $\mathrm{s}$ & $\phi$ & Função phase-field & $(-)$ \\
\hline Operação transposta da matriz & $(-)$ & \multicolumn{3}{|c|}{ Sub índices } \\
\hline Vetor velocidade & $\mathrm{m} / \mathrm{s}$ & $o$ & Óleo & \\
\hline Componente de velocidade & & $w$ & Água & \\
\hline na direção z & & & & \\
\hline
\end{tabular}

\section{REFERÊNCIAS}

ANDRADE, G. M. Análiese termo-fluido-dinâmica de escoamento bifásico óleo-água em tubulações compostas de multicamadas com aquecimento elétrico ativo. 137p. (Mestrado).

COPPE, Engenharia Oceânica, Universidade Federal do Rio de Janeiro, Rio de Janeiro. 2009.

BIRD, R. B., W. E. STEWART, et al. Transport phenomena. Nova York: 2002.

CAHN, J. W. and J. E. HILLIARD. Free energy of a nonuniform system. I. Interfacial free energy. The Journal of Chemical Physics, v. 28, p. 258-267, 1958.

CHEN, F. and P. D. YAPA. Three-Dimensional Visualization of multi-phase (oil/gas/hydrate) plumes. Environmental Modelling \& Software, v. 19, p. 751-760, 2004.

COMSOL (2013). COMSOL Multiphysics 4.3a Documentation.

GIRAULT, V. and P. A. RAVIART. Finite element approximation of the Navier-Stokes equations. Lecture Notes in Mathematics, Berlin Springer Verlag, v. 749, p. 208, 1979.

HENNING, C. D. Oil/gas collector/separator for underwater oil leaks. United States, Patent, 1993. 
MARIANO, G. C. Estudo do escoamento imiscível água/óleo mediante experimentação em célula de Hele-Shaw e simulação CFD. 85p. (Mestrado). Pós-Graduação em Engenharia Química, Universidade Federal de Santa Catarina, Florianólis. 2008.

MIRANDA, J. Consequencias do vazamento de petróleo no Golfo do México. Grupo Escolar 2011. Disponível em: < http://www.grupoescolar.com/pesquisa/consequencias-do-vazamentode-petroleo-no-golfo-do-mexico.html >. Acesso em: 30/01/2011.

PALADINO, E. E. Modelagem matemática e simulação numérica de trajetórias de derrames de petróleo no mar. 110p. (Mestrado). Engenharia Mecânica, Universidade Federal de Santa Catarina, Florianópolis. 2000.

ROSSI, L. F. d. S. and R. E. MORALES. Modelagem matemática do escoamento de emulsão envolvendo água e óleo. Instituto brasileiro de petróleo e gás, v. 3, p. 9, 2004.

SANTOS, R. G. d., R. S. MOHAMED, et al. Avaliação da molhabilidade de oleodutos através de medidas de ângulo de contato: efeitos de asfaltenos e ácidos naftênicos do petróleo. 105p. (Mestrado). São Paulo. 2003.

WÖRNER, M. A compact introduction to the numerical modeling of multiphase flows. Forschungszentrum Karlsruhe, 2003.

YAPA, P. D., L. ZHENG, et al. Modeling Underwater Oil/Gas Jets and Plumes. Journal of Hydraulic Engineering, v. 125, p. 481, 1999.

YUE, P., C. Zhou, et al. Phase-field simulations of interfacial dynamics in viscoelastic fluids using finite elements with adaptive meshing. Journal of Computational Physics, v. 219, p. 4767, 2006. 